\title{
Multicentre phase II trial of trastuzumab and capecitabine in patients with HER2 overexpressing metastatic pancreatic cancer
}

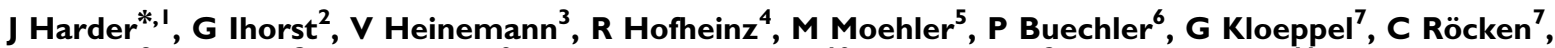 M Bitzer ${ }^{8}$, S Boeck ${ }^{3}$, E Endlicher', A Reinacher-Schick ${ }^{10}$, C Schmoor ${ }^{2}$ and M Geissler ${ }^{11}$}

'Medizinische Klinik II, Hegau- Bodensee Klinikum, Virchowstraße 10, D-78224 Singen, Germany; ${ }^{2}$ Clinical Trials Unit, University Medical Center Freiburg, Elsässer Straße 2, D-79I 10 Freiburg, Germany; ${ }^{3}$ Klinikum Grosshadern Medizinische Klinik III, L.M.- Universitätsklinikum München, Marchoninistraße I5, D-8I 377 München, Germany; ${ }^{4}$ Medizinische Klinik III, Klinikum Mannheim, Theodor-Kutzer-Ufer I-3, D-68I67 Mannheim, Germany; ${ }^{5}$ Medizinische Klinik und Poliklinik, Klinikum der Johannes Gutenberg- Universität Mainz, Langenbeckstraße I, D-55 I 3 I Mainz, Germany; ${ }^{6}$ Abt. Allgemeine,- Viszerale-u. Unfallchirurgie, Chirurgische Universitätsklinik Heidelberg, Im Neuenheimer Feld I 10, D-69I 20 Heidelberg, Germany; 'Institut f. Pathologie, ChristianAlbrechts-Universität, Arnold Heller-Straße 3, D-24I 05 Kiel, Germany; ${ }^{8}$ Medizinische Klinik I, Universitätsklinikum Tübingen, Ottfried-Müller- Straße 10 , D-72076 Tübingen, Germany; ${ }^{9}$ Klinik und Poliklinik für Innere Medizin I, Klinikum der Universität Regensburg, Franz-Josef-Strauss-Allee II, D-93042 Regensburg, Germany; ${ }^{10}$ Hämatologie/Onkologie, Knappschaftskrankenhaus Bochum, In der Schornau 23-25, D-44892 Bochum, Germany; "'Klinik für Allg. Innere Medizin, Onkologie/Hämatologie, Gastroenterologie u. Infektiologie, Klinikum Esslingen, Hirschlandstraße. 97, D-73730 Esslingen, Germany

BACKGROUND: New therapeutic options for metastatic pancreatic cancer are urgently needed. In pancreatic cancer, overexpression of the epidermal growth factor receptor 2 (HER2) has been reported in up to 45\%. This multicentre phase II study investigated the efficacy and toxicity of the HER2 antibody trastuzumab combined with capecitabine in the patients with pancreatic cancer and HER2 overexpression.

METHODS: Primary endpoint was progression-free survival (PFS) after 12 weeks. A total of 212 patients were screened for HER2 expression.

RESULTS: Immunohistochemical (IHC) HER2 expression was: 83 (40\%) grade 0, 7 I (34\%) grade I, 3I (I 5\%) grade 2, 22 (I I\%) grade 3. A total of 17 patients with $1 \mathrm{HC}+3$ HER2 expression or gene amplification could be assessed for the treatment response. Grade $3 / 4$ treatment toxicities were: each 7\% leucopenia, diarrhoea, nausea and hand-foot syndrome. Progression-free survival after 12 weeks was $23.5 \%$, median overall survival (OS) 6.9 months.

CONCLUSION: This study demonstrates +3 HER2 expression or gene amplification in I I\% of patients. Contrary to breast and gastric cancer, only 7 out of I I (64\%) patients with $H \mathrm{HC}+3$ HER2 expression showed gene amplification. Although the therapy was well tolerated, PFS and OS did not perform favourably compared with standard chemotherapy. Together, we do not recommend further evaluation of anti-HER2 treatment in patients with metastatic pancreatic cancer.

British Journal of Cancer (2012) 106, 1033-1038. doi:I0.1038/bjc.2012.18 www.bjcancer.com

Published online 28 February 2012

(C) 2012 Cancer Research UK

Keywords: pancreatic cancer; immunohistochemistry; growth factors; chemotherapy

Pancreatic cancer has a very poor prognosis, making it one of the five most common causes of cancer mortality in developed countries. After curative intended resection only $5-10 \%$ of patients with adenocarcinoma of the pancreas will be alive at 5 years after diagnosis. Advanced (unresectable locally advanced or metastatic) pancreatic cancer is an incurable disease with limited treatment options.

Since more than a decade, the nucleoside analogue gemcitabine is regarded as a standard of care for patients with advanced disease, providing clinical benefit and a moderate improvement in survival (Burris et al, 1997). Several randomised phase III trials failed to show a survival benefit for gemcitabine-based combination chemotherapy so far; however, meta-analytic data suggest a possible survival benefit for the use of fluoropyrimidines including capecitabine in combination with gemcitabine

*Correspondence: Dr J Harder; E-mail: jan.harder@hbh-kliniken.de Received 7 July 20 I I; revised 6 January 20 12; accepted 12 January 20 I2; published online 28 February 2012 in selected patients, that is, those with metastatic disease and a good performance status (Heinemann et al, 2008). The anti-EGFR tyrosine kinase inhibitor erlotinib was demonstrated to result in superior survival, especially in patients developing skin rash (Moore et al, 2007). HER2 is a related receptor tyrosine kinase encoded by proto-oncogenes. Once activated, the signaltransduction cascade promotes cellular proliferation migration and survival. Immunohistochemical (IHC) overexpression of HER2 in various tumour cells has been not only associated with a poor prognosis, but also offers the therapeutic option of receptor targeting therapies. Studies report HER2 expression in up to $45 \%$ of patients with pancreatic cancer (Yamanaka et al, 1993). Targeting HER2 in pancreatic cancer cell lines and a xenograft mouse model showed encouraging results (Buechler et al, 2001, 2005).

Therefore, the aim of this study was to clarify the clinical significance of HER2 expression in patients with metastatic pancreatic cancer and to determine the potential of HER2 as therapeutic target in these patients. 
On the basis of these data, we assessed the activity of the combination of trastuzumab and capecitabine in patients with advanced IHC + 3 HER2 expressing pancreatic cancer or HER2 gene amplification. As the outcome of patients with locally advanced and metastatic pancreatic cancer is different and the response assessment in the latter is more reliable, only patients with metastatic disease were included. The objectives of the trial were to determine progression-free survival (PFS) after 12 weeks (primary endpoint), PFS, overall survival (OS), response rate (according to RECIST criteria), clinical benefit response (CBR) and safety profile.

\section{PATIENTS AND METHODS}

\section{Patients and treatment}

This was a prospective, single-arm, open-label, multi-centre phase II trial to investigate the efficacy and safety of trastuzumab (Herceptin) and capecitabine (Xeloda) as first-line treatment in patients with IHC +3 HER2 expressing advanced pancreatic cancer or cancer with HER2 gene amplification of stage IVB $\left(\mathrm{T}_{1-4} \mathrm{~N}_{0-1} \mathrm{M}_{1}\right)$.

Patients' inclusion and exclusion criteria are shown in Table 1. Patients fulfilling the main inclusion and exclusion criteria were screened for HER2 expression. Patients were included into the trial in case of IHC +2 HER2 expression (additionally confirmed by gene amplification, fluorescence in situ hybridisation (FISH)) or +3 HER2 expression.

Patients received $4 \mathrm{mg} \mathrm{kg}^{-1}$ trastuzumab at first infusion followed by weekly $2 \mathrm{mg} \mathrm{kg}^{-1}$ combined with $1250 \mathrm{mg} \mathrm{m}^{-2}$ capecitabine twice daily on days $1-14$ of a 3 -week cycle. Treatment was continued until disease progression.

The study was approved by the institutional review board and ethics committee of each participating centre, and informed consent was given by each patient according to the Declaration of Helsinki. The trial is registered with WHO primary register number DRKS00000600.

\section{Procedures}

Immunohistochemistry Immunohistochemistry was performed on adjacent deparaffinised freshly cut sections using the peroxidase-labelled streptavidin-biotin technique, Dako REAL detection system (Glostrup, Denmark) for HER2. All immunostaining was performed in strict accordance with the FDA-approved REAL detection system package (Dako). Immunohistochemical results were scored independently by two pathologists 'blind' to all case data. Additional tissue controls were performed along with the included cell line controls.

Detection of HER2 was performed with heat-induced epitope retrieval and the use of the anti-HER2 primary antibody. Immunohistochemical staining was performed using the Dako Autostainer. The percentage of carcinoma cells was estimated in categories negative, weak $(1+)$, moderate $(2+)$ or intense $(3+)$ if $10 \%$ or more of carcinoma cells attain plasma membrane staining. Cytoplasmic staining was discounted.

Fluorescence in situ hybridisation The PathVysion detection kit (Abbott Laboratories, Abbott Park, IL, USA) was used for FISH analysis in case of IHC +2 HER2 expression. Previous studies showed in breast and colorectal cancer but also in biliary malignancies, which are biologically related to pancreatic cancer, that +3 HER2 expression is regular induced via gene amplification (Kobayashi et al, 2002; Ooi et al, 2004; Carlson et al, 2006; Yau et al, 2008; Harder et al, 2009). Therefore, we did not perform FISH analysis in +3 HER2 expressing tumour specimens before treatment with trastuzumab.

FISH-stained sections were scanned at $\times 1000$ magnification and three separate carcinoma areas identified. Twenty nuclei were assessed in each area; HER 2 and chromosome 17 copy numbers were counted for all cells and the ratio of HER2 to chromosome 17 was calculated. Normal mean HER2: 17 ratio was defined as $<2$; a ratio $>2$ was taken as gene amplification. Normal mean HER2 copy number was taken as $<4$ signals per cell.

In order to explain the treatment results we retrospectively analysed 11 out of 17 tumour specimens for HER2 gene amplification from patients with IHC +3 HER2 expression who were treated by trastuzumab and capecitabine. Six could not be analysed due to various reasons (mainly cytological samples or to small sample size).

The retrospective testing used the HER2-SISH double labelling in situ hybridisation system, and the Ventana BenchMark XT automated slide staining system (all Roche Diagnostics $\mathrm{GmbH}$, Mannheim, Germany). Gene amplification was assessed according to the breast cancer scoring system (Rüschoff et al, 2009).

\section{Endpoints}

Tumour response was evaluated by CT or MRI every 2 cycles (every 6 weeks) and classified as complete remission (CR), partial remission (PR), stable disease, progressive disease (PD) according to RECIST (Therasse et al, 2000).

Primary endpoint was the PFS rate 12 weeks after the start of treatment. Secondary endpoints were PFS time, OS time, time

Table I Inclusion and exclusion criteria

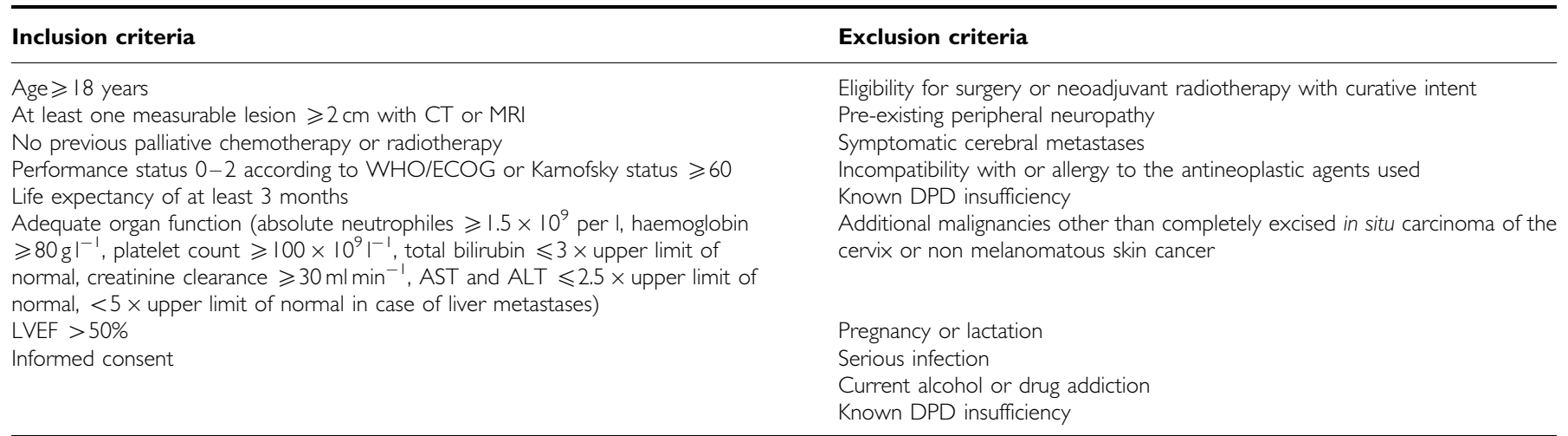

Abbreviations: $A L T=$ alanine aminotransferase; $A S T=$ aspartate aminotransferase; $C T=$ computed tomography; DPD = dihydropyrimidindehydrogenase; $E C O G=$ Eastern Cooperative Oncology Group; LVEF = left ventricular ejection fraction; MRI = magnetic resonance imaging; WHO=World Health Organization. 
to response (CR/PR), duration of response, CBR 12 weeks after the start of treatment and quality of life (QOL) using the EORTC QLQ-C30 QOL Questionnaire. Clinical benefit response is a composite endpoint assessing the improvement in pain (pain intensity and analgesic consumption) and Karnofsky performance status as primary measures and integrating body weight as a secondary measure. Patients were classified as responders, if pain or Karnofsky performance status was improved or, in case of stability of pain and Karnofsky performance status, body weight was increased (Burris et al, 1997).

PFS was defined as the time from beginning of chemotherapy to disease progression or death, whichever occurred first. Overall survival time was defined as the time from beginning of chemotherapy to death.

Toxicity was evaluated according to NCI/CTC version 3.0 of 12 December 2003.

\section{Statistical analysis}

Sample size calculation was based on the primary endpoint PFS rate 12 weeks after the start of treatment. The number of patients to be included was determined by the two-stage design according to Simon (1989) and was based on the following considerations. After monotherapy with capecitabine, median PFS time is 2.8 months (Cartwright et al, 2002). On the basis of these results, treatment with trastuzumab and capecitabine is considered to be not sufficiently active if the PFS rate 12 weeks after the start of treatment is $50 \%$ or lower. Treatment with trastuzumab and capecitabine is considered to be promising for further evaluation if the PFS rate 12 weeks after start of treatment is $70 \%$ or higher.

In a first step, 23 patients should be included and treated in the study. If 12 or less of these 23 patients were alive and free of PD after 12 weeks, the study should be stopped and the treatment considered ineffective in this group of patients. If at least 13 patients were alive and free of progression after 12 weeks of treatment, recruitment should be continued until 37 patients were included. If 24 or more of these 37 patients were alive and free of progression after 12 weeks, treatment should be considered as promising and should be studied further. This procedure ensures that if the treatment has a true PFS rate of at least $70 \%$, the chance of erroneously rejecting the regime is $\leqslant 20 \%$. The chance of erroneously considering the treatment effective is $\leqslant 5 \%$ if the true PFS rate is $\leqslant 50 \%$.

The primary efficacy analysis was based on the full analysis set (FAS), including all patients for whom treatment was started. An additional analysis was performed in the per protocol (PP) population with those patients who had received at least two complete cycles of chemotherapy, or had terminated treatment due to toxicity, early progression or death before day 43 . The PFS rate and the OS rate were estimated by the Kaplan-Meier method. The effect of CA 19-9 serum concentration was investigated with a Cox regression model. Here, a cutpoint of $1000 \mathrm{U} \mathrm{ml}^{-1}$ for CA 19-9 was chosen as value close to the median $796 \mathrm{U} \mathrm{ml}^{-1}$ in order to obtain two patient groups of approximately the same size.

The safety analysis set (SAF) was defined as those patients who received at least one dose of chemotherapy. The SAF is identical to the FAS in this study, therefore no further distinction will be made. The incidence of toxicity and adverse events (AEs) was calculated as the number of patients who experienced at least one toxicity/AE of a certain category as a percentage of the total number of patients.

SAS software (SAS Institute Inc., Cary, NC, USA) version 9.2 was used for the analysis.

\section{RESULTS}

Between July 2004 and May 2008, a total of 212 patients were screened for HER2 expression and eligibility criteria at nine institutions. In 207 patients, the tumour specimens could be assessed for HER2 expression and gene amplification. In IHC $83(40 \%)$ were grade $0,71(34 \%)$ grade $1,31(15 \%)$ grade 2 and $22(11 \%)$ grade 3 , respectively. One tumour specimen with IHC grade 2 showed gene amplification. In the initial assumption of the study protocol all IHC grade 3-positive specimens were considered FISH positive (HER2 to chromosome 17 ratio $>2$ ) and treated with trastuzumab if there were no other exclusion criteria. In a post hoc analysis, taking into account the low response rates, we analysed 11 out of 17 IHC +3 HER2 expressing samples from patients who were treated with trastuzumab and capecitabine for HER2 gene amplification. Seven tested positive (64\%) and four were negative.

Of 23 patients with IHC + 3 HER 2 expression or IHC + 2 HER2 expression and HER2 gene amplification, $11(47.8 \%)$ had a CA 19-9 value at screening $\geqslant 1000 \mathrm{U} \mathrm{ml}^{-1}$, whereas among 163 patients without HER2 gene amplification, 74 (45.4\%) CA 19-9 values were $<1000 \mathrm{U} \mathrm{ml}^{-1}$.

The study was closed prematurely due to low HER2 expression and slow recruitment after screening 212 patients, 97 women (45.8\%); median age 64 years, range 38-86 (Table 2).

From the 23 patients with IHC +3 HER 2 expression or IHC +2 and HER2 gene amplification, 17 patients from four centres were included in the study and could be assessed for response to treatment in the full analysis set, 8 women (47.1\%); median age 64 years, range 42-77 (Table 1). Five patients were not included because of violated inclusion/exclusion criteria. One patient was not included in the trial for other reasons (Figure 1).

Patients received a median number of three cycles (range 1-23), a median weekly trastuzumab dose of $145 \mathrm{mg}$ (range 91-330), and a median weekly capecitabine dose of $15,429 \mathrm{mg}$ (range $4500-$ 31250 ), relating to median $99.4 \%$ of the planned trastuzumab dose

Table 2 Baseline patient characteristics

\begin{tabular}{|c|c|c|}
\hline & $\begin{array}{l}\text { Patients } \\
\text { screened } \\
(N=212)\end{array}$ & $\begin{array}{l}\text { Patients } \\
\text { included } \\
(N=17)\end{array}$ \\
\hline $\begin{array}{l}\text { Age at registration (years): median } \\
\text { (range) }\end{array}$ & $64(38-86)$ & $64(42-77)$ \\
\hline \multicolumn{3}{|l|}{ Sex: n (\%) } \\
\hline Female & $97(45.8)$ & $8(47.1)$ \\
\hline \multicolumn{3}{|l|}{ Tumour stage at first diagnosis: $\mathrm{n}(\%)$} \\
\hline I & $3(1.6)$ & $0(0)$ \\
\hline$\|$ & $16(8.7)$ & $2(11.8)$ \\
\hline III & $60(32.6)$ & $4(23.5)$ \\
\hline IV & $47(25.5)$ & $7(41.2)$ \\
\hline Not evaluable & $58(31.5)$ & $4(23.5)$ \\
\hline Not available & 28 & - \\
\hline \multicolumn{3}{|l|}{ Adenocarcinoma: n (\%) } \\
\hline Yes & $202(98.1)$ & $17(100)$ \\
\hline No & $4(1.9)$ & 0 \\
\hline Not available & 6 & - \\
\hline \multicolumn{3}{|l|}{ IHCIFISH: n (\%) } \\
\hline 0 & $83(40.1)$ & 0 \\
\hline $1+$ & $71(34.3)$ & 0 \\
\hline $2+/ \mathrm{FISH}-$ & $30(14.5)$ & 0 \\
\hline $2+/ \mathrm{FISH}^{+}$ & I $(0.5)$ & । (5.9) \\
\hline $3+$ & $22(10.6)$ & $16(94.1)$ \\
\hline Not available & 5 & - \\
\hline $\begin{array}{l}\text { CA 19-9 }\left(\cup \mathrm{ml}^{-1}\right) \text {, median (range); } \\
\text { not available: } n=22\end{array}$ & $766(0-190,953)$ & $796(8-130,603)$ \\
\hline
\end{tabular}

Abbreviations: $\mathrm{CA}=$ carbohydrate antigen; $\mathrm{FISH}=$ fluorescence in situ hybridisation; $\mathrm{IHC}=$ immunohistochemistry 


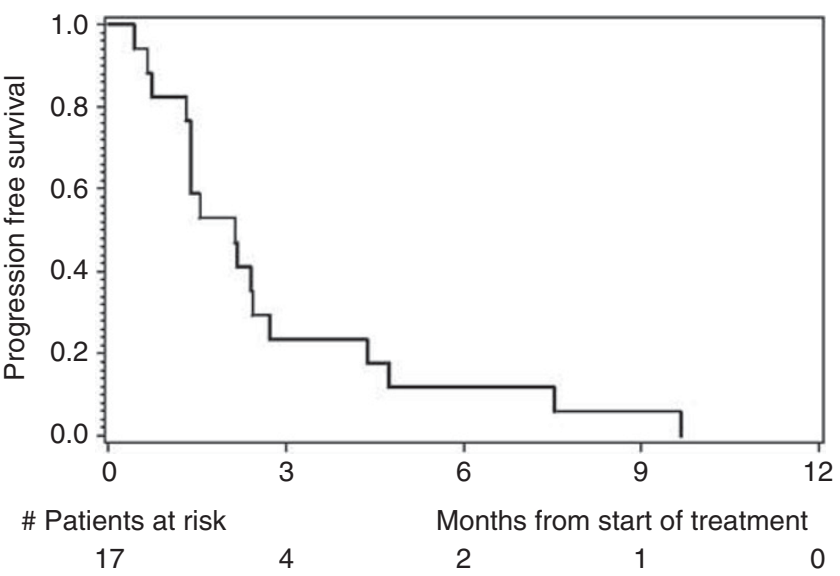

Figure 2 Kaplan-Meier estimates of progression-free survival.

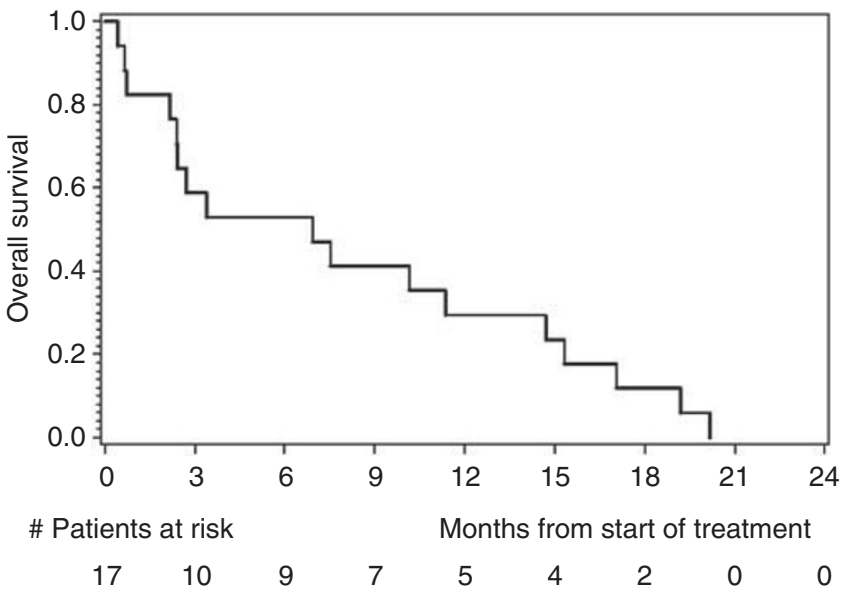

Figure 3 Kaplan-Meier estimates of overall survival.

A total number of 101 AEs were observed, relating to an average number of 5.9 AEs per patient, range $0-17$. Of these, 16 AEs were considered as serious (SAE). Most frequent AEs were of gastrointestinal origin (12 out of $17,70.6 \%$ ), general health problems as fatigue, (9 out of $17,52.9 \%$ ) and infections in 6 out of 17 patients $(35.3 \%)$.

\section{DISCUSSION}

The expression of the growth factor receptors HER2 has been studied in different tumour types leading to the standard therapeutic use in breast cancer. Recently trastuzumab in combination with chemotherapy was considered as a new standard option for patients with HER2-positive advanced gastric or gastro-oesophageal junction cancer (Bang et al, 2010).

In other cancers drugs such as trastuzumab and lapatinib are under investigation. There are data of HER2 expression in up to $45 \%$ of patients with pancreatic cancer in mainly small cohorts (Yamanaka et al, 1993). Therefore, this study was designed to investigate the combination of trastuzumab and capecitabine as palliative first-line therapy in patients with metastatic pancreatic cancer. Beside the report by Yamanaka et al, there are two reports by Safran et al $(2001,2004)$ who first screened 154 patients with pancreatic cancer and showed HER2 overexpression in $21 \%$ (Safran et al, 2001) and 16\% (Safran et al, 2004) of patients, respectively. Thirty-four patients were treated with trastuzumab 
and gemcitabine because the tumours showed HER2 overexpression ( +2 HER2 in 30 and +3 HER2 in 4 tumours (Safran et al, $2004)$ ). From the $21 \%$ of 154 patients (Safran et al, 2001), 23 (15\%) had IHC + 2 HER2 expressing tumours and $9(6 \%)+3$ HER2 expression, examined by standardised methods. In 3 out of 8 $(40 \%)$ IHC +2 HER 2 and 0 out of $3+3$ HER 2 expressing tumours they could show gene amplification by FISH. In the second cohort, they report HER2 amplification in 2 out of 13 and hyperploid chromosome 17 in 3 out of 13 specimens with IHC HER2 overexpression.

This multi-centre study demonstrates IHC +2 HER2 expression in 31 out of 207 (15\%) and + 3 HER2 expression in 22 out of 207 $(11 \%)$ of patients with metastatic pancreatic cancer. Only 7 out of $11(64 \%)$ patients with IHC + 3 HER2 expression showed gene amplification and 1 out of 22 (5\%) with +2 HER expression.

The data for IHC HER2 expression reported by Safran et al and from this study are comparable except for HER2 gene amplification in IHC +2 HER2 expressing tumours. One explanation therefore might be the different test method used for the first cohort reported by Safran et al (2001). In the second cohort they summarised gene amplification for IHC +2 and +3 HER2 expression and counted hyperdiploid chromosome 17 as FISH positive, irrespective of the ratio HER2 to chromosome 17. Second they (Safran et al, 2004) used CT-guided biopsies or fine-needle aspirates, whereas in our study most samples were true cut biopsies. The sample quality might be important especially for FISH. These two points might explain the difference in FISH positivity between current series and Safran et al especially in IHC +2 HER2 expressing tumours. In summary, reports of HER2 expression, not only in pancreatic cancer, are confusing as the term 'overexpression' was also used for + 1 HER2 immunostaining (Yamanaka et al, 1993). As the example from HER2 IHC shows there is a strong need for consistent and accurate reporting of biomarker data.

Breast, colorectal and biliary cancer shows high concordance between HER2 expression and amplification (Ooi et al, 2004; Yau et al, 2008; Harder et al, 2009). Furthermore, HER2 amplification is associated with treatment response (Hudis, 2007). In gastric cancer a new histological scoring system for HER2 IHC was established in the ToGA trail (Bang et al, 2010). Additional HER2 gene amplification was found in $13 \%$ and $27 \%$ in IHC +1 and +2 tumours, respectively, although almost all gastric tumours with IHC + 3 HER2 expression showed gene amplification.

In a post hoc analysis, we performed FISH for gene amplification in +3 HER2 expressing tumours in order to explain the conflicting data regarding HER2 amplification in IHC + 2 HER2 tumours and the poor treatment results found in this study. In accordance with Safran et al this study shows no consistent HER2 gene amplification in IHC +3 expressing pancreatic adenocarcinoma as seen in other cancers, suggesting some other pathways resulting in protein overexpression. Contrary to breast, colon, biliary and gastric cancer in pancreatic cancer IHC +2 HER2 expression and especially + 3 HER2 expression does not correlate with gene amplification. May be HER2 overexpression in pancreatic cancer is due to gene deregulation rather than gene amplification as postulated by Ukita et al (2002) for intrahepatic biliary tract cancer.

Due to the low incidence of IHC + 3 HER2 expression, only 17 patients could be treated with trastuzumab and capecitabine in this trial. Although the therapy was well tolerated, and PFS and OS are comparable to previous regimens, the combination of trastuzumab and capecitabine did not perform favourably compared with historical standard gemcitabine or capecitabine chemotherapy.

The RR reported by Safran et al (2004) was $24 \%$ overall and even $34 \%$ for patients with HER2 gene amplification resulting in a median OS of 7.5 months. These encouraging results led to our study design and numbers calculated. Gemcitabine is considered as the chemotherapy of choice for metastatic pancreatic cancer.
There is no comparative trial between Gemcitabine and Capecitabine, but Capecitabine has to be considered to have superior response rates over bolus 5-FU and similar to Gemcitabine (Burris et al, 1997; Cartwright et al, 2002). Therefore, we designed this study in intention for a patient-friendly protocol including an oral chemotherapy. In addition there was the hope that combination therapy including capecitabine might result in better response rates taking into account the disappointing results using gemcitabine in combination with anti-growth factor therapies (Xiong et al, 2004). Median PFS in our trial was 65 days with an OS of 6.9 month, which is similar to single-agent capecitabine (Cartwright et al, 2002). In a recent study, published in abstract form (McDermott et al, 2011) capecitabine in combination with lapatinib, a small molecule, tyrosine kinase inhibitor of epidermal growth factor receptor and HER2 showed disappointing antitumour activity with an OS of 4 months. Our study showed an median OS of 6.9 months, but taken together capecitabine in combination with anti-HER2 therapy does not seem to significantly improve treatment results in comparison with historical capecitabine monotherapy (mean OS 6.0 months; Cartwright et al, 2002). Therefore, a comparative trial can not be recommended.

That one-third of IHC $3+$ HER2-positive tumours showed no HER2 amplification found in a post hoc analysis might be one explanation for the disappointing treatment results adding antiHER2 treatment to capecitabine. The phenomenon that targeted therapy containing regimens are ineffective in pancreatic cancer while the same regimens have shown significant activity in other GI tumour entities is also known for anti-EGFR and anti-VEGF drugs (Kindler et al, 2010; Philip et al, 2010). Therefore, it is also unlikely that the combination of trastuzumab and cetuximab will show synergistic effects in human pancreatic cancer as suggested by a xenograft mouse model (Larbouret et al, 2010). There may be downstream signalling events involved in non-responsiveness to trastuzumab such as RAF, PI3K including the presence of k-ras mutations, which can be found in $60-70 \%$ of pancreatic cancer patients. In addition, IGF and or TGFb signalling may overcome the effect of HER2 targeting (Vaccaro et al, 2011). Additional, tumour-associated fibroblasts and stromal development may counteract the effect of chemotherapy including targeted therapy. The heterogeneous and complex biology of pancreatic cancer or some specific tumour-stroma interaction, in a cancer with a strong desmoplastic environment, might are some reasons why many chemotherapy regimens containing an anti-growth factor inhibitor failed to significantly improve OS. According to recent reports one promising way to overcome chemotherapy resistance in pancreatic cancer is to focus on the tumour environment and tumour stem cells (Bednar and Simeone, 2011; Liles et al, 2011).

There are limitations of this study. First, we miscalculated the needed study sites for rapid patient recruitment as shown by Bang et al for gastric cancer. Therefore, the study had to be closed prematurely. Second, erroneous we did not perform FISH for IHC +3 HER2 expressing tumours prospectively. Relying on the data found for breast, colon and biliary cancer we assumed HER2 amplification in +3 HER 2 expressing tumours. Third, there was no control group comparing anti-HER2 treatment with conventional chemotherapy because the study was designed as a two-step phase II study. This way testing chemotherapy regiments with only a few study sites does not fit for biomarker-guided trials studying targeted therapy applicable to $10-20 \%$ of patients.

Despite these limitations, we can conclude that $\mathrm{IHC}+2$ and +3 HER2 expression is present in about $25 \%$ but HER2 gene amplification in only $4 \%$ of patients with pancreatic cancer. Even in patients with HER2 amplifying tumours, the combination chemotherapy with trastuzumab and capecitabine does not result in improved PFS and OS compared with historical gemcitabine or capecitabine alone. According to these results we do not recommend further evaluation of anti-HER2 treatment in patients with metastatic pancreatic cancer. 


\section{REFERENCES}

Bang YJ, Van Cutsem E, Feyereislova A, Chung HC, Shen L, Sawaki A, Lordick F, Ohtsu A, Omuro Y, Satoh T, Aprile G, Kulikov E, Hill J, Lehle M, Rüschoff J, Kang YK, ToGA Trial Investigators (2010) Trastuzumab in combination with chemotherapy versus chemotherapy alone for treatment of HER2-positive advanced gastric or gastrooesophageal junction cancer (ToGA): a phase 3, open-label, randomised controlled trial. Lancet 376: 687-697

Bednar F, Simeone DM (2011) Pancreatic cancer stem cell biology and its therapeutic implications. J Gastroenterol 46: 1345-1352

Buechler P, Reber HA, Buechler MC, Roth MA, Buechler MW, Friess H, Isacoff WH, Hines OJ (2001) Therapy for pancreatic cancer with a recombinant humanized anti HER2 antibody (herceptin). J Gastrointest Surg 5: $139-146$

Buechler P, Reber HA, Eibl G, Roth MA, Buechler MW, Friess H, Isacoff WH, Hines OJ (2005) Combination therapy for advanced pancreatic cancer using Herceptin plus chemotherapy. Int J Oncol 27: $1125-1130$

Burris H, Moore MJ, Andersen J, Green MR, Rothenberg ML, Modiano MR, Cripps MC, Portenoy RK, Storniolo AM, Tarassoff P, Nelson R, Dorr FA, Stephens CD, Von Hoff DD (1997) Improvements in survival and clinical benefit with gemcitabine as first-line therapy for patients with advanced pancreas cancer: a randomized trial. J Clin Oncol 15: $2403-2413$

Carlson RW, Moench SJ, Hammond ME, Perez EA, Burstein HJ, Allred DC, Vogel CL, Goldstein LJ, Somlo G, Gradishar WJ, Hudis CA, Jahanzeb M, Stark A, Wolff AC, Press MF, Winer EP, Paik S, Ljung BM (2006) NCCN HER2 Testing in Breast Cancer Task Force. HER2 testing in breast cancer: NCCN Task Force report and recommendations. J Natl Compr Canc Netw 4(Suppl 3): S1 -22; quiz S23-S24

Cartwright TH, Cohn A, Varkey JA, Chen YM, Szatrowski TP, Cox JV, Schulz JJ (2002) Phase II study of oral capecitabine in patients with advanced or metastatic pancreatic cancer. J Clin Oncol 20: 160-164

Harder J, Waiz O, Otto F, Geissler M, Olschewski M, Weinhold B, Blum HE, Schmitt-Graeff A, Opitz OG (2009) EGFR and HER2 expression in advanced biliary tract cancer. World J Gastroenterol 15: $4511-4517$

Heinemann V, Boeck S, Hinke A, Labianca R, Louvet C (2008) Metaanalysis of randomized trials: evaluation of benefit from gemcitabinebased combination chemotherapy applied in advanced pancreatic cancer. BMC Cancer 8: 82

Hudis CA (2007) Trastuzumab- mechanism of action and use in clinical practice. $N$ Engl J Med 357: 39-51

Kindler HL, Niedzwiecki D, Hollis D, Sutherland S, Schrag D, Hurwitz H, Innocenti F, Mulcahy MF, O'Reilly E, Wozniak TF, Picus J, Bhargava P, Mayer RJ, Schilsky RL, Goldberg RM (2010) Gemcitabine plus bevacizumab compared with gemcitabine plus placebo in patients with advanced pancreatic cancer: phase III trial of the Cancer and Leukemia Group B (CALGB 80303). J Clin Oncol 28: 3617-3622

Kobayashi M, Ooi A, Oda Y, Nakanishi I (2002) Protein overexpression and gene amplification of c-erbB-2 in breast carcinomas: a comparative study of immunohistochemistry and fluorescence in situ hybridization of formalin-fixed, paraffin-embedded tissues. Hum Pathol 33: 21-28

Larbouret C, Robert B, Bascoul-Mollevi C, Penault-Llorca F, Ho-PunCheung A, Morisseau S, Navarro-Teulon I, Mach JP, Pèlegrin A, Azria D (2010) Combined cetuximab and trastuzumab are superior to gemcitabine in the treatment of human pancreatic carcinoma xenografts. Ann Oncol 21: $98-103$

Liles JS, Arnoletti JP, Kossenkov AV, Mikhaylina A, Frost AR, Kulesza P, Heslin MJ, Frolov A (2011) Targeting ErbB3-mediated stromal-epithelial interactions in pancreatic ductal adenocarcinoma. $\mathrm{Br} J$ Cancer 105: $523-533$
McDermott RS, Calvert P, Parker M, Webb G, Moulton B, McCaffrey J (2011) A phase II study of lapatinib and capecitabine in first-line treatment of metastatic pancreatic cancer (ICORG 08- 39). J Clin Oncol 29(Suppl. 4): Abstract 315

Moore MJ, Goldstein D, Hamm J, Figer A, Hecht JR, Gallinger S, Au HJ, Murawa P, Walde D, Wolff RA, Campos D, Lim R, Ding K, Clark G, Voskoglou-Nomikos T, Ptasynski M, Parulekar W, National Cancer Institute of Canada Clinical Trials Group (2007) Erlotinib plus gemcitabine compared with gemcitabine alone in patients with advanced pancreatic cancer. A phase III trial of the National Cancer Institute of Canada Clinical trials group. J Clin Oncol 25: 1960-1966

Ooi A, Takehana T, Li X, Suzuki S, Kunitomo K, Iino H, Fujii H, Takeda Y, Dobashi Y (2004) Protein overexpression and gene amplification of HER-2 and EGFR in colorectal cancers: an immunohistochemical and fluorescent in situ hybridization study. Mod Pathol 17: 895-904

Philip PA, J Moinpour CM, Vaught NL, Goldman B, Redman MW, Millwood B, Lippman SM, Seay TE, Flynn PJ, O'Reilly EM, Rowland KM, Wong RP, Benedetti J, Blanke CD (2010) Pain and emotional well-being outcomes in Southwest Oncology Group-directed intergroup trial S0205: a phase III study comparing gemcitabine plus cetuximab versus gemcitabine as first-line therapy in patients with advanced pancreas cancer. J Clin Oncol 28: $3605-3610$

Rüschoff J, Nagelmeier I, Hofmann M, Henkel T, Stoss O (2009) ErbB2 diagnostics in breast cancer-an update. Pathologe 30(2): 147-155

Safran H, Iannitti D, Ramanathan R, Schwartz JD, Steinhoff M, Nauman C, Hesketh P, Rathore R, Wolff R, Tantravahi U, Hughes TM, Maia C, Pasquariello T, Goldstein L, King T, Tsai JY, Kennedy T (2004) Herceptin and gemcitabine for metastatic pancreatic cancers that overexpress HER2/neu. Cancer Invest 22: 706-712

Safran H, Steinhoff M, Mangray S, Rathore R, King TC, Chai L, Berzein K, Moore T, Iannitti D, Reiss P, Pasquariello T, Akerman P, Quirk D, Mass R, Goldstein L, Tantravahi U (2001) Overexpression of the HER-2/neu oncogene in pancreatic adenocarcinoma. Am J Clin Oncol 24: 496-499

Simon R (1989) Optimal two-stage designs for phase II clinical trials. Control Clin Trials 10: $1-10$

Therasse P, Arbuck SG, Eisenhauer EA, Wanders J, Kaplan RS, Rubinstein L, Verweij J, Van Glabbeke M, van Oosterom AT, Christian MC, Gwyther SG (2000) New guidelines to evaluate the response to treatment in solid tumors. European Organization for Research and Treatment of Cancer, National Cancer Institute of the United States, National Cancer Institute of Canada. J Natl Cancer Inst 92: 205-216

Ukita Y, Kato M, Terada T (2002) Gene amplification and mRNA and protein overexpression of c-erbB-2 (HER-2/neu) in human intrahepatic cholangiocarcinoma as detected by fluorescence in situ hybridization, in situ hybridization and immunohistochemistry. J Hepatol 36: 780-785

Vaccaro V, Melisi D, Bria E, Cuppone F, Ciuffreda L, Pino MS, Gelibter A, Tortora G, Cognetti F, Milella M (2011) Emerging pathways and future targets for the molecular therapy of pancreatic cancer. Expert Opin Ther Targets 15: $1183-1196$

Yamanaka Y, Friess H, Kobrin MS, Buechler M, Kunz J, Beger HG, Korc M (1993) Overexpression of HER2/neu oncogene in human pancreatic carcinoma. Hum Pathol 24: 1127-1134

Yau TK, Sze H, Soong IS, Hioe F, Khoo US, Lee AW (2008) HER2 overexpression of breast cancers in Hong Kong: prevalence and concordance between immunohistochemistry and in-situ hybridisation assays. Hong Kong Med J 14: 130-135

Xiong HQ, Rosenberg A, LoBuglio A, Schmidt W, Wolff RA, Deutsch J, Needle M, Abbruzzese JL (2004) Cetuximab, a monoclonal antibody targeting the epidermal growth factor receptor, in combination with gemcitabine for advanced pancreatic cancer: a multicenter phase II Trial. J Clin Oncol 22: 2610-2616

This work is published under the standard license to publish agreement. After 12 months the work will become freely available and the license terms will switch to a Creative Commons Attribution-NonCommercial-Share Alike 3.0 Unported License. 